\title{
Adaptación al cambio climático: un análisis desde la gobernanza y el capital social al caso de la comuna de Frutillar - Chile
}

\author{
Adaptation to climate change: an analysis from governance and \\ social capital to the case of the commune of Frutillar - Chile
}

\section{Karla V. Romero Baeza}

\section{Resumen}

Las particularidades de las condiciones climáticas ambientales actuales, en particular de aquellas que impactan en los territorios, generan la necesidad de desarrollar estrategias de adaptación a nivel local, especialmente para la reducción de riesgos y la protección medioambiental. La literatura afirma que este proceso puede ser favorecido por las caracterices del capital social y la gobernanza local en los territorios locales. Este estudio explora las relaciones entre el capital social, la gobernanza y la adaptación al cambio climático en la comuna de Frutillar, región de Los Lagos, Chile. A través de un modelo mixto de diseño exploratorio y el uso de técnicas de recolección de información en base a documentación oficial, entrevistas, grupo de discusión y técnicas de análisis de contenido combinadas con procesamiento de datos, se lograron obtener resultados que sugieren la existencia de debilidades en las estrategias de adaptación desplegadas en la comuna de Frutillar debido a la priorización de solo medidas de mitigación. El estudio concluye afirmando que las estrategias de adaptación no solo se consiguen con buena voluntad o con instrumentos de planificación, es necesario el desarrollo de prácticas efectivas desplegadas desde y hacia el territorio. Sin aquello, la adaptación al cambio climático no será adecuada ni valiosa para los habitantes del territorio.

\section{Palabras claves}

Gobernanza Local; Capital Social; Cambio Climático; Estrategias de Adaptación.

\section{Abstract}

The particularities of the current environmental climate conditions, specifically those that impact the territories, generate the need to develop adaptation strategies at the local level. The literature states that this process can be favored by the characteristics of social capital and local governance in local territories, especially for risk reduction and environmental protection. This study explores the relationships between social capital, governance and adaptation to climate change in the commune of Frutillar, Los Lagos region, Chile. Through a mixed model of exploratory design and the use of information gathering techniques based on official documentation, interviews, discussion group and content analysis techniques combined with data processing. It was possible to obtain results that suggest the existence of weaknesses in the adaptation strategies deployed in the commune of Frutillar due to the prioritization of only mitigation. The study concludes stating that adaptation strategies are not only achieved with goodwill or planning instruments, it is necessary to develop effective practices from and to the territory. Without that, adaptation to climate change will not be adequate or valuable for the inhabitants of the territory.

\section{Keywords}

Local Governance; Social Capital; Climate Change; Adaptation Strategies. 


\section{Introducción ${ }^{1}$}

Las variaciones del clima son un aspecto normal en los ciclos naturales de la tierra (IPCC, 2007; IPCC, 2014), pero desde finales del siglo XX hay una creciente preocupación global. Por tal razón, iniciativas globales como el Sistema de Naciones Unidas (ONU), el Panel Intergubernamental del Cambio Climático (IPCC), la Organización de las Naciones Unidas para la Alimentación y la Agricultura (FAO), han documentado exhaustivamente el cambio climático, sus impactos y sus estrategias de adaptación.

Chile no está ajeno. En el país existen territorios más vulnerables al cambio climático (ABDÓN y MEZA, 2008, p. 8). Por su parte, la Comisión Económica para Latinoamérica y el Caribe (CEPAL, 2008) informa que la mayor parte del país está expuesto a los efectos del cambio climático, éstos estarían en torno al aumento de las temperaturas, baja densidad de precipitaciones, aridez de los suelos en zonas de cultivo, inundaciones, por nombrar algunos (CEPAL, 2008; CONAMA, 2006; VALDIVIESO, ANDERSSON y VILLENA-ROLDÁN, 2017). Fenómenos que tendrían impacto en la pesca, minería, agricultura, salud, bosques, ganadería, llevando a los territorios a modificar sus dinámicas económicas y sociales existentes con el medio natural (IPCC, 2007; IPCC 2014; CEPAL, 2008; CONAMA, 2006).

Ahora bien, ¡cómo lograr que territorios y comunidades vulnerables a los efectos nocivos del cambio climático y eventos extremos desarrollen estrategias que les permitan una adecuada adaptación? La literatura es bien clara: los procesos de adaptación son el resultado de las decisiones individuales, sociales e institucionales (GALINDO, SAMANIEGO, ALATORRE y FERRER, 2014; VALDIVIESO, 2016; VALDIVIESO y ANDERSSON, 2017; VALDIVIESO et al. 2017; ANDERSSON y VALDIVIESO, 2018). Asimismo, el conocimiento del tema, la educación, la superación de la pobreza, la participación en redes, el acceso a programas sociales y la participación social son factores que permiten adaptase al cambio climático adecuadamente (GALINDO, SAMANIEGO, ALATORRE y FERRER, 2013). Entonces, la adaptación es el resultado de decisiones humanas, sociales e institucionales, y son las nociones de capital social y las relaciones de gobernanza los temas que abordaremos en este estudio en particular.

\footnotetext{
${ }^{1}$ La autora agradece las recomendaciones de dos árbitros anónimos, más las del editor de este número de Revista Debates, ya que permitieron mejorar la versión final del estudio. Asimismo, agradece el apoyo financiero que hizo posible las actividades de investigación para elaborar este estudio: Proyectos Fondecyt Conicyt no 1140672 y no 1181282.
} 
La gobernanza será entendida como un proceso estructurado mediante el cual diversos actores públicos, privados y sociales, se coordinan eficiente y eficazmente en el despliegue de políticas consensuadas en pro de un objetivo de intereses público, donde aquella coordinación puede ser tanto a/desde escala local hacia otras escalas como nacionales o internacionales o viceversa (RHODES, 1996; AGUILAR, 2007; BLANCO y GOMÀ, 2003; NATERA, 2004; COMISIÓN EUROPEA, 2001; WHITTINGHAM, 2010; VALDIVIESO, 2016; VALDIVIESO y ANDERSSON, 2017; VALDIVIESO et al. 2017; ANDERSSON y VALDIVIESO, 2018). Mientras que el Capital Social consistirá en recursos individuales y colectivos basados en interacciones, relaciones de confianza, acuerdos y reciprocidad que una vez movilizados permiten obtener retornos que contribuyen a alcanzar objetivos individuales como colectivos (PUTNAM, 1993 y 2000; VALDIVIESO y VILLENAROLDÁN, 2012 y 2014; VALDIVIESO y DAVIDOVICS, 2016). Esos recursos son función de distintos factores que afectan las decisiones individuales y colectivas, tales como las características de los individuos o el impacto que tienen las instituciones públicas en las personas y sus organizaciones (OSTROM, 2008; ROSHTEIN y STOLLE, 2008; USLANER, 2003; VALDIVIESO y DAVIDOVICS, 2016).

Observar aquellos componentes en territorios específicos es fundamental para generar estrategias y acciones orientadas a una mejor adaptación al cambio climático. En el caso de Chile, lo anterior es particularmente importantes, porque en la escala local no se ven progresos homogéneos (VALDIVIESO, 2016 y 2017).

En este artículo, el territorio de estudio la comuna de Frutillar en la Región de Los Lagos. Donde nos preguntaremos ¿existen respuestas locales y en qué consisten?, ¿por qué se desarrollan de la forma en que lo hacen?, ¿hay un sistema de gobernanza que apoye la adaptación?, ¿̧hay capital social que apoye la adaptación?, en definitiva explorar si hay relaciones entre el capital social, la gobernanza y la adaptación al cambio climático. Por lo tanto, el objetivo general del estudio consiste en comprender la relación entre gobernanza y capital social como factores explicativos de las estrategias de adaptación al cambio climático y eventos extremos en la comuna de Frutillar. La elección de dicha comuna se debió al hecho de ser representativa de las comunas medianas y pequeñas de Chile, por lo expuesta que está al cambio climático, y la creciente necesidad por estrategias que contribuyan a un mayor bienestar de la población.

El estudio se basó en un diseño exploratorio de tipo mixto. El diseño metodológico incluyó el uso de diversas técnicas de recolección de información, como 
lo fueron: análisis bibliográfico, utilización de fuentes secundarias, entrevistas en profundidad, entrevistas semiestructuradas y grupos de discusión. Donde cada uno de éstos responden a protocolos diseńados por los proyectos Fondecyt n. 1140672 y n. 1181282, en cuya adaptación para aplicación en la comuna de Frutillar trabajó la autora de este artículo. Los temas consultados en las entrevistas fueron, en relación con las respuestas de adaptación, los siguientes: (1) experiencias personales con el cambio climático y eventos extremos, y efectos sociales y económicos de éste; (2) respuestas y acciones de las municipalidades y otras instituciones, y valoración de esas actividades; (3) participación social y capacidad de organización de la población, tipos de organizaciones sociales, frecuencia de reuniones, niveles de confianza entre personas, organizaciones sociales y el rol que cumplen en la comuna.

El trabajo de campo se realizó en distintas etapas. En la primera se realizaron entrevistas en profundidad a: Director de fundación PLADES, concejal de la comuna, dirigente social del sector urbano, dirigente social del sector rural y profesional encargado de PRODESAL. En una segunda parte, a partir de mayo 2014, se aplicaron entrevistas semiestructuradas a 17 jefes de hogar. Posteriormente, en julio de 2014 se realizó un grupo de discusión con el propósito de validar las informaciones recabadas en las dos primeras etapas, asistieron 25 personas donde destacan: dirigentes sociales, funcionarios municipales y concejales. Entre junio y julio de 2014 se realizaron las entrevistas semi-estructuradas a 3 directivos de la Municipalidad de Frutillar, de los departamentos de obras, emergencias y planificación comunal. Adicionalmente en agosto de 2014 se realizó taller con equipo municipal del Programa PRODESAL en dependencias del Centro de Estudios del Desarrollo Regional (CEDER) de la Universidad de Los Lagos, Osorno con el objetivo de conocer las estrategias de intervención municipal en el sector rural. Estas entrevistas fueron complementadas con nuevas entrevistas y consultas tanto a funcionarios municipales como a dirigentes sociales durante los años 2016 y 2018.

Durante el período de la investigación, en paralelo a las actividades de campo, se recopiló la información de fuentes secundarias de instrumentos de planificación local y regional, tales como: zonificación de riesgos ambientales de Frutillar (2014), Plan Urbano Estratégico Frutillar, Cuentas Públicas 2012, 2013 y 2014, Plan de Desarrollo Comunitario 2007 - 2012 y 2015 - 2018, Estrategia de Desarrollo Regional de Los Lagos (2008-2020), Plan Maestro de áreas verdes de Frutillar, presupuestos del gobierno local de salud, educación y municipal con sus correspondientes modificaciones, notas de prensa, actas de concejo municipal entre 
enero 2011 a mayo 2014, y estadísticas secundarias obtenidas con información proporcionada por el Instituto Nacional de Estadísticas.

$\mathrm{El}$ análisis de contenido de toda la información cualitativa fue procesado con el apoyo del programa de análisis de contenido ATLAS-TI. Con el programa SPSS fue procesada la información cuantitativa, con el propósito de obtener estadísticas descriptivas.

Este artículo está estructurado de la siguiente manera. En un primer lugar se plantea una revisión de literatura en torno al cambio climático, la gobernanza y el capital social. En segundo lugar, se da cuenta del impacto del cambio climático en Chile y en sus regiones y las consiguientes medidas de adaptación a nivel estatal, regional y local. En tercer lugar, se dan a conocer los resultados en torno a las características de la gobernanza y capital social en la comuna de Frutillar. En cuarto lugar, se discutirán las relaciones entre esos conceptos y la adaptación. Finalmente, se darán a conocer las conclusiones y desafíos en torno al objeto de estudio.

\section{Cambio climático, ecologia y adaptación}

¿Será variabilidad climática o Cambio Climático? El IPCC (2007) señala que la variabilidad del clima es una variación de las estadísticas en torno a un promedio, considerándose una característica propia del clima, en cambio, el cambio climático es un modificación en los patrones climáticos en el largo plazo, donde en los últimos 100 ańos la temperatura global subió $0,74^{\circ}$ en la superficie del planeta. El informe del año 2001 define al cambio climático como una "importante variación estadística en el estado medio del clima o en su variabilidad, que persiste durante un período prolongado (normalmente decenios o incluso más)" (IPCC, 2001, p. 175). Donde éste se puede deber a procesos naturales internos o a cambios del forzamiento externo, o bien a cambios persistentes antropogénicos en la composición de la atmósfera o en el uso de las tierras (IPCC, 2001; IPCC 2007; IPCC, 2013).

Diversos estudios documentan una serie de impactos de éste sobre los sistemas naturales y humanos, siendo aquello reforzado por los informes del IPCC (1990, 2001, 2007 y 2013). Los impactos son entendidos como las externalidades positivas o negativas de ciertos factores en el medio natural y su entorno social. Tales manifestaciones pueden verse en áreas como la agricultura, los bosques y el ecosistema, los recursos hídricos, la salud humana, las industrias y la sociedad. Cada evento extremo del clima acarrea efectos bastante nocivos para cada sector señalado. Por un lado, la agricultura, los bosques y los ecosistemas se ven afectados por el daño 
en la flora y fauna, y existiendo incluso un aumento de plagas. Por otro lado, existirá déficit hídrico como la contaminación de éste. En tanto, las consecuencias urbanas estarán en torno a procesos de migración migración, déficit de agua, perdidas de la calidad de vida de las personas, problemas en la industria y la alimentación (IPCC, 2007; 2013; FAO, 2009). Entonces, cada uno de estos efectos pondrá en merma los procesos de desarrollo de los territorios, generando la urgencia de estrategias innovadoras que permitan la adaptación de manera más eficiente a los efectos del cambio climático y eventos extremos.

\section{Gobernanza y buen gobierno local, elementos para su análisis}

Las municipalidades y los gobiernos locales cumplen funciones relevantes para el desarrollo territorial de las comunas en la escala local (VALDIVIESO 2016; ANDERSSON y VALDIVIESO, 2018). Por sus mandatos legales y su cercanía a los problemas locales, ellas tienen una posición privilegiada para promover el desarrollo, fortaleciendo los procesos de gobernanza local (NATERA, 2004). Por esta razón, la literatura de la gobernanza reconoce el importante papel de las instituciones locales, y propone que las relaciones de proximidad entre distintos actores, a distintas escalas, favorecen el buen gobierno (por ejemplo, BLANCO y GOMÀ, 2003). Este concepto ha influido en la investigación empírica en las Ciencias Sociales, principalmente frente a la gestión eficiente de la política en los territorios y la gestión medioambiental.

Gobernanza es un proceso que cuenta con estructuras, donde los actores políticos llevan a cabo prácticas de intercambio, coordinación y adopciones de decisiones en sistemas democráticos, donde el gobierno de manera horizontal gestiona las redes y organizaciones de naturaleza múltiple (NATERA, 2004). A su vez es un juego de poderes con reglas e instituciones formales como informales (WHITTINGHAM, 2010; COMISIÓN DE LAS COMUNIDADES EUROPEAS, 2001). De este modo, permite la politización del espacio local ante problemas comunes (BLANCO y GOMÀ, 2003).

La gobernanza integra distintas escalas de aplicación, por lo mismo, la gobernanza local y multinivel no son excluyentes entre sí, los buenos resultados dependen de que el sistema local trabaje adecuadamente con otras escalas de gobernanza, tales como agencias de gobiernos, organizaciones internacionales, entre otras. Debido a la existencia de incentivos, la capacidad de los sistemas de gobernanza local descansa en las condiciones para el aprendizaje interactivo, que facilita los ajustes 
de las reglas y comportamientos para tener respuestas efectivas (VALDIVIESO, 2016).

El proceso de gobernanza favorece la cooperación de actores operando en distintas escalas, contribuyendo a procesos de toma de decisión más eficientes y eficaces que los desarrollados por sistemas centralizados. En contextos de estados centralizados, como es el caso de Chile, la gobernanza es un proceso que implica descentralización y entrega de poder por parte del Estado central hacia otros actores, sean estatales de escalas territoriales locales $\mathrm{u}$ actores sociales privados, ONGs u empresas (RHODES, 1996; VELÁZQUEZ, 2005; AGUILAR, 2007; VALDIVIESO, 2012; BLANCO y GOMÀ, 2003; NATERA, 2004; COMISIÓN EUROPEA, 2001; WHITTINGHAM, 2010).

Considerando que los municipios como institución local tienen una posición privilegiada para la gestión de las redes de su territorio, VALDIVIESO (2016 y 2017) y VALDIVIESO y ANDERSSON (2017) proponen aspectos claves para el análisis: la cantidad de interacciones y las frecuencias de éstas, la participación en distintas escalas y los flujos de información que existen. Entendiendo que los procesos de gobernanza y los buenos resultados se ven favorecidos cuando hay mayor cantidad de interacciones, existen adecuados flujos de información y procesos de aprendizaje que enriquecen las decisiones de los gobiernos locales.

En definitiva, algunos de los elementos para analizar los procesos de gobernanza en los territorios están dados claramente y resumidos en los aportes entregados anteriormente. Sin embargo es fundamental que la aplicación teórica del concepto no sea una receta, puesto que la gobernanza en sí misma es compleja y opera de modo distinto, según el contexto territorial.

\section{Nociones en torno al capital social}

No existe un consenso respecto a la conceptualización del capital social, los abordajes teóricos son distintos según perspectiva teórica de los investigadores, sus estratégicas metodológicas, las fuentes de información disponibles y las técnicas de estimación (VALDIVIESO, 2012; VALDIVIESO y VILLENA-ROLDÁN, 2012; VALDIVIESO y DAVIDOVICS, 2016). En este apartado serán sintetizadas propuestas que tienen el potencial de enriquecer la investigación empírica en contextos territoriales.

Coleman (1998) lo precisa como un vínculo entre el actor social con la estructura social, entendiéndose que los recursos estructurales son un recurso activo 
para los individuos que facilitan acciones comunes para quienes forman parte de la estructura. Esser (2008) lo conceptualiza en relación con la posición de un individuo, distinguiendo la existencia de dos niveles: uno relacional y otro sistémico. El primero es aquél donde ego se relaciona con actores sociales que tienen recursos que le permiten obtener información útil para tomar decisiones, siendo ego el principal beneficiario del intercambio. El capital social relacional se divide a su vez en un capital social basado en la posición, en la confianza o en las obligaciones. La idea del capital social sistémico parte del supuesto que las personas tienen necesidades e intereses comunes que dan origen a una organización social - una red de relaciones-, y aquella voluntad de construcción se fortalece a través de un sistema de control efectivo, donde cada uno de los integrantes contribuye a la organización, y los vínculos de la organización representan algo más que los individuos. Por su otra parte, Ahn y Ostrom (2008) lo relacionan con la acción colectiva, postulan que los actores sociales movilizan recursos que generan una inversión tanto individual como colectiva, donde el capital social es entendido como los recursos que se producen y que ayudan a resolver dilemas y conflictos que contribuyen a tomar decisiones más inteligentes, estando compuesto por: confianza social y arreglos institucionales. Complementariamente, Lin (2008) propone que éste es una inversión con recursos de valor. Los individuos invierten al establecer vínculos con otras personas, puesto que poseen una expectativa de movilizar los recursos que tiene la organización para conseguir sus propios objetivos. Roshtein y Stolle (2008) destacan que el capital social no se produce solo por lógicas individuo-comunidad, sino por el impacto que tienen las instituciones públicas en las personas y sus organizaciones. Uslaner (2003) complementa diciendo que la confianza en las instituciones tiene efectos en la confianza social de las personas y que ésta se produciría a través de gobiernos transparentes y abiertos a la participación de la comunidad en el mismo.

Entonces estipulativamente entenderemos al capital social para los efectos de nuestra propia investigación como recursos individuales y colectivos, basados en relaciones de confianza y reciprocidad, que una vez movilizados permiten obtener retornos que contribuyen a alcanzar objetivos individuales como comunitarios. El cual puede ser producido tanto por lógicas individuales como por el impacto que tienen las instituciones públicas en las personas y sus organizaciones (PUTNAM, 1993 y 2000; VALDIVIESO, 2012; VALDIVIESO y VILLENA-ROLDÁN, 2012; VALDIVIESO y DAVIDOVICS, 2016: HARTMUT, 2008; OSTROM, 2008; LIN, 2008; ROSHTEIN y STOLLE, 2008; USLANER, 2003). 


\section{Chile, escenarios posibles ante el Cambio Climático y marco regulatorio de adaptación}

Por las condiciones geográficas propias del país, existen territorios más expuestos a eventos extremos del clima que otros. (CEPAL, 2008; ABDÓN y MEZA, 2008; CONAMA, 2006; IPCC, 2013; VALDIVIESO et al., 2017). El estudio de Conama (2006) señala que en Chile considerando escenarios A2 y B2 existirá un aumento de la temperatura, en el A2 el incremento sería entre $4^{\circ}$ y $5^{\circ}$, mientras que en el B2 estaría $1^{\circ}-2^{\circ}$ y $2^{\circ}-3^{\circ}$. En cuanto a las precipitaciones, en el sector norte grande se prevé un aumento de éstas, donde en el escenario A2 el aumento llegará a la I región y en el B2 a la II región, en el norte chico también se pronostica un aumento de las precipitaciones anuales, mientras que para las zonas del Chile central y zona sur se estima que las precipitaciones disminuirían, en la zona sur aquello estaría entre un $25 \%$ en primavera a un $40 \%$ en verano, y en la zona austral alcanzará un $25 \%$. Por otra parte, el aumento del nivel de mar, que tiene un alto impacto en el contexto nacional será un proceso que se evidenciará en cambios de entre $10 \mathrm{~cm}$ y $20 \mathrm{~cm}$, dependiendo de la altitud de la región (CEPAL, 2008, p. 7).

En Chile existe plan nacional de adaptación vigente: el Plan Nacional de Adaptación al Cambio Climático (PNACC) 2014 del Ministerio del Medio Ambiente, describe una serie de acciones transversales y acciones sectoriales. Las primeras son: investigación científica que debe definir un marco de referencia científico, fomentar investigaciones del área y divulgación científica; comunicación y educación ambiental, definiendo una estrategia comunicacional y generando educación y sensibilización ambiental; fortalecimiento institucional y reducción del riesgo de desastres que genere información para la toma de decisiones en el marco de la gestión del riesgo de desastres. Las segundas consisten en la elaboración de planes en los siguientes sectores: silvoagropecuario, recursos hídricos, biodiversidad, pesca y acuicultura, salud, energía, infraestructura, ciudades y turismo; consulta ciudadanas de los planes e implementación de los mismos. Además contempla un monitoreo constante de cada una de las líneas de acción para la adaptación. Recientemente, los gobiernos chilenos han comenzado incluir a las municipalidades como actores relevantes en el proceso de adaptación (VALDIVIESO, 2016; VALDIVIESO y ANDERSSON, 2017).

En el contexto de la Región de Los Lagos, donde se ubica la comuna de Frutillar (Figura 1), se destaca que habrá una disminución sustancial de las 
precipitaciones, en el escenario B2 el descenso será de un 30\%, mientras que en el A2 será más pronunciada la disminución, llegando a un 40\% (CEPAL, 2008).

Figura 1 - Frutillar (4107’17,81” S - 7303’37,72” O), Región de Los Lagos - Chile

Fuente: Biblioteca del Congreso Nacional de Chile.

También se espera una disminución de los caudales hidrográficos tanto por el efecto de la disminución de las precipitaciones como por el aumento de las temperaturas. El aumento del nivel del mar es un fenómeno que ocurrirá, pero los efectos que provocaran en la región no es posible describirlos puesto que no existen estudios empíricos al respecto. Por último, los suelos de la región se verán altamente erosionados, aumentando el riesgo de inundaciones (CEPAL, 2008). Ante tales proyecciones climáticas, CEPAL (2008) estima una serie de impactos económicos positivos y negativos en la Región, que en líneas generales son: Aumento superficie cultivos, aumento superficie dedicada a frutales, decaimiento superficies de praderas naturales, aumento superficies forestales y plagas: hipotéticamente, sin sustento empírico, podría existir una disminución de plagas de hongos por el aumento de las temperaturas. Sin embargo, tal aumento podría provocar en que insectos amplíen sus nichos ecológicos y de por si aumenten su población.

En el caso de la comuna de Frutillar, la información proporcionada tanto por sus habitantes como por fuentes secundarias consultadas sugiere que los efectos del 
cambio climático se están haciendo día a día más visibles, y afectan las condiciones de vida de los habitantes. Estas circunstancias, combinadas con particularidades geográficas y peligros de desastres naturales, tales como los riesgos derivados de la cercanía al Volcán Osorno y al Volcán Calbuco, y la dependencia de sus sectores productivos con el medio ambiente, hacen que este territorio sea vulnerable al cambio climático y eventos extremos.

Si bien, la Región de Los Lagos y Frutillar cuentan con características propias que podrían permitir sacar ventajas de los efectos del cambio del clima, es necesario progresar en estrategias de adaptación antes las cambiantes circunstancias del escenario medioambiental.

\section{Adaptación Cambio Climático, gobernanza local y capital social en Frutillar}

Según la información proporcionada por diagnósticos e instrumentos de planificación territorial local, como lo son: la Zonificación de riesgos socioambientales 2013, el Plan de Desarrollo Comunitario (PLADECO) 2015 2018, el Plan de emergencias comunal, y un Plan Urbano Estratégico Frutillar 2013, Frutillar contaría con infraestructura de calidad suficiente para definirse a sí misma como una comuna con enfoque en el desarrollo sustentable, en el PLADECO de la Municipalidad de Frutillar 2015 - 2018 afirma que tiene:

\footnotetext{
Un gobierno local organizado y orientado a los vecinos, quienes son el centro del quehacer, entregando bienes y servicios de excelencia mediante una administración innovadora y que busca alcanzar el desarrollo de la comuna sustentable, viable y equitativa, en donde los ciudadanos participan respecto a sus decisiones, se conectan e interactúan y generan valor a su territorio (MUNICIPALIDAD DE FRUTILLAR, PLADECO, 2018, p. 94).
}

Ahora bien, las modificaciones climáticas están teniendo sentido para sus habitantes, un 82,6\% de los jefes de hogar entrevistados en este estudio (17 entrevistados) señalaron que se percibe un cambio del clima en la comuna desde la última década, donde los eventos más recurrentes fueron la sequía, las inundaciones y las lluvias extremas. Algunos testimonios afirmaron que:

"No sabemos en qué época sembrar, porque ya el tiempo no es como antes, hay heladas en estaciones que antes no habían, también llueve en épocas 
que hacía calor (grupo de discusión, 24 de julio de 2014). Asimismo, Por el clima provoca que por la sequía o inundaciones los cultivos se suben o se pudren" (Grupo de discusión, 24 de julio de 2014).

Siendo la sequía, para un $65 \%$ de los entrevistados, uno de los mayores eventos climáticos que los afectan. Los funcionarios municipales tienen conciencia que la comuna está siendo afectada y son necesarias las acciones coordinadas. El Encargado de Emergencia de la municipalidad informó que:

"La sequía que hubo acá fue fuerte, la que te conté. Hace unos años donde nosotros llegamos con cambiones aljibes que nos donó el gobierno regional que se destinaban a través de bomberos. Tenían una capacidad de 10.000 litros de agua, con ese camión abastecíamos, y cuándo no podíamos arrendábamos camiones a través del municipio con presupuesto municipal y estábamos llegando con 20.000 litros de agua, ese fue nuestro plan de contingencia, volvíamos tarde tipo 10.00 de la noche pero logramos dejar a la gente contenta, trabajamos en un plan de poder abastecer con estanques de agua con su llavecita y todo para que la gente tuviera, entonces teníamos estanques con logos del gobierno que dejábamos en los sectores, en cada casa y nosotros llenábamos estanques" (Entrevista, 16 de julio de 2014).

Ahora bien, la pregunta es ¿Serán siempre así de reactivas las acciones desarrolladas por la municipalidad frente a adversidades producidas por el cambio climáticos y/o eventos extremos? La revisión de actas del concejo municipal (desde enero de 2011 a mayo de 2014) dejó en evidencia la recurrencia de dichas prácticas, se menciona:

Se solicita limpieza cuneta Ruta 5 en el sector de La Huacha, Concejal Eladio Rivera - Sesión Ordinaria No 20 del 12.07.2011 en archivo”, "Calle Bernales, existen viviendas que están al mismo nivel que la calle provocando que en épocas de lluvias la vivienda se inunda, plantea que solución puede ser que exista drenaje en el patio, pero Alcalde señala que hay que verlo con Director de Obras, Concejal Luis Espinosa - Sesión Ordinaria No 07 del 04.03.2013 en archivo", "Existe la importancia de drenar los campos por el tema de las inundaciones, Concejal César Huenuqueo - Sesión Ordinaria No 23 del 19.08.2013 en archivo (MUNICIPALIDAD DE FRUTILLAR, 2014). 
Por otra parte, los instrumentos de gestión comunal mencionados en párrafos anteriores solo señalan medidas de mitigación en el mejor de los casos. La municipalidad está comenzando a dar sus primeros pasos en la planificación medioambiental. La Directora de la Secretaria Comunal de Planificación (SECPLAN) informó:

\footnotetext{
"Estoy encargada del PMG (Programa de Mejoramiento de la Gestión Municipal), de generar nuevas prácticas internas, hacer un catastro de las energías que se están usando, calefactores y gasto de agua. Ahora estamos en el primer año de diagnóstico y aprendizaje, que es el medio ambiente, y todo eso lo apoya el ministerio y financia todo lo que son los talleres y al final del proceso hay que construir como un comité social con las organizaciones sociales para después hacer el levantamiento comunal y también sé que hay un par de colegios que ya están certificados por el sello medioambiental, pero ahora el municipio está como recién partiendo en la etapa de diagnóstico, por lo menos en el PMG también se enmarca en lo que es el Cambio Climático" (Entrevista, 8 de julio de 2014).
}

Claramente se advierte que el municipio está comenzando recién a sensibilizarse sobre temáticas ambientales, y las respuestas de adaptación al cambio climático, por tanto, suelen ser de tipo reactivas.

Lo anterior fue confirmado por otros funcionarios municipales consultados (3 entrevistados). Respecto a actividades de prevención de desastres naturales el 100\% señaló que no se generan estrategias de educación ambiental, en cambio un 66,7\% estableció que si se realizan actividades de comunicación para prevenir eventos extremos. Los mismos exponen que la información proporcionada a la comunidad la extraen de internet o estudios de expertos en materia ambiental, en la misma índole, al consultar sobre la influencia de aquella información en la planificación comunal, en una escala del 1 al 5, un 66,7\% manifestó que tiene 4 de influencia. Entonces, solo se están priorizando actividades de comunicación para la prevención ante eventos extremos antes que la educación ambiental. Aunque claramente se observan esfuerzos por parte del municipio para realizar intervenciones comunicativas y de planificación contextualizadas en las particularidades de la comuna.

$\mathrm{Al}$ contrario, las entrevistas realizadas a habitantes y dirigentes sociales de la comuna, sugieren que la municipalidad no es una fuente de información relevante. Al ser consultados sobre el acceso a información durante eventos climáticos extremos, las 
personas entrevistadas mencionaron en un $91,3 \%$ que se informan por la radio como primer medio, como segundo medio un $52,2 \%$ señaló que utiliza internet y un $34,8 \%$ mencionó que se informa a través del municipio. Claramente, el municipio no está siendo considerado como el principal medio de información. Durante el encuentro con dirigentes de organizaciones sociales (25 participantes), los discursos fueron negativos hacia la gestión municipal y demuestran desconformidad con las prácticas para transmitir información, algunas personas mencionaron:

"Las autoridades no nos dan información de cómo prevenir desastres en los campos (grupo de discusión, 24 de julio de 2014), No sabemos en caso de crisis a quién acudir, a funcionarios de organismos regionales o provinciales (grupo de discusión, 24 de julio de 2014) y Tienen nulo conocimiento de facilidades para enfrentarse a la crisis" (Grupo de discusión, 24 de julio de 2014).

En relación a planes municipales y relaciones de gobernanza, el Encargado de la Oficina de Emergencias informó:

"Planes de respuestas existen. Siempre estamos preparados para poder
resolver con nuestros propios medios primero y después cuando estamos
colapsados tenemos que pedir apoyo a la gobernación provincial o a la
intendencia regional que es nuestro último escalafón. Después de la
intendencia se piden los recursos a nivel nacional por medio del
Ministerio del Interior" (Entrevista, 16 de julio de 2014).

La respuesta es clara, las primeras acciones son locales siempre y cuando la situación no se ve superada, si ven que los esfuerzos no son suficientes, se sienten en la obligación de solicitar recursos y apoyos a nivel provincial, regional o nacional. En el caso de la sequía, tal como se mencionó en párrafos anteriores, la estrategia de mitigación es realizada en múltiple colaboración y coordinación con la Oficina Nacional de Emergencia (ONEMI), alcalde, gobierno provincial, gobierno regional, bomberos, actores del mundo privado como Patagonia Global y grupos organizados de ciudadanos como son las Juntas de vecinos. Al respecto, los directivos municipales mencionaron en un $100 \%$ que existe colaboración con distintas reparticiones gubernamentales para enfrentar desastres naturales causados por el clima. Con respecto a las posibilidades presupuestarias para planificar o realizar actividades de prevención un $100 \%$ de los entrevistados manifestó que esto influye en las estrategias que pueden o no desarrollar. A su vez, ellos valoran positivamente los recursos 
económicos recibidos de las institucionales de las distintas escalaras territoriales, sin embargo, un $66,7 \%$ de los consultados sienten que aquello contribuye a implementar actividades y/o políticas que están decididas fuera del municipio. Pese a existir una red de convergencias la autonomía del gobierno local se ve truncada por las disposiciones políticas que tienen las escalas regionales y nacionales.

Tal como se mencionó más atrás, no solo una red de gobernanza adecuada posibilita la adaptación. Otro factor relevante es el capital social en el territorio. En Frutillar existen 430 organizaciones sociales y territoriales (MUNICIPALIDAD DE FRUTILLAR, 2018). Los propósitos son variados, siendo algunos: obtención y/o mejoramiento de viviendas, clubes de adulto mayor, clubes deportivos y rurales, por nombrar algunos. Además desde el año 2012, por aplicación de la Ley 20.500 se conforma el Consejo Comunal de las Organizaciones de la Sociedad Civil (COSOC).

Los arreglos institucionales o las obligaciones reciprocas que resultan de las relaciones sociales reiteradas y la confianza pueden ser relevantes para la resiliencia y la adaptación al cambio climático. Al consultar a los jefes de hogar (17 entrevistados) sobre si existen grupos de personas que se movilicen cuando existe un problema, un $52,2 \%$ de los consultados señaló que no existen dichos grupos. Mientras que un $60,9 \%$ mencionó que la ayuda en tales momentos depende de la voluntad de las personas más que de un acuerdo formal de alguna organización.

Otros elementos del capital social son las relaciones de reciprocidad, los flujos de información y la efectividad de alcanzar propósitos. Un 69,6\% de los entrevistados señaló que participa en una organización para tener información y solucionar un problema, pero solo un $39,1 \%$ de éstos manifestó que aquello contribuyó a la solución del problema en cuestión, respecto a la concreción de metas solo un 47,8\% señaló que las organizaciones las alcanzan.

En relación a las relaciones regulares de una organización, un 78,3\% señaló que se congregan frecuentemente, pero según el 69,6\% de los entrevistados solo llegan pocos a las reuniones. Situación que cambia cuando asiste una autoridad, puesto que un $65,2 \%$ de los consultados manifestó que cuando ésta llega a la reunión asisten más asociados que lo habitual.

Se observa que existe una expectativa de participación cuando asisten autoridades municipales a las reuniones de las organizaciones. Un 69,6\% de los entrevistados mencionó que hay relación con el municipio y/u otros servicios públicos, donde en la mayoría de los casos es para comunicar algún problema de la comunidad. Donde además un $82,6 \%$ de los consultados considera que la 
organización que debiese coordinarse con las autoridades frente a problemas comunes son las juntas de vecinos del territorio.

\section{Estrategias de adaptación en el territorio de Frutillar ante el cambio climático}

Los habitantes de Frutillar, como los funcionarios públicos de la municipalidad advierten que el clima está cambiando y éstos últimos reconocen que es necesario el desarrollo de estrategias para su enfrentamiento. Políticas públicas nacionales existen pero no se evidencia un impacto de éstas en lo local. Si se aprecian planes comunales y esfuerzos de sus funcionarios por realizar acciones que tiendan a la mitigación de los eventos climáticos extremos, donde ellos mismos están conscientes de que aún no realizan actividades de educación y sensibilización ambiental. Los funcionarios públicos del municipio dan cuenta que la comunicación, como estrategia de prevención de desastres para ellos es prioritaria. Si bien el municipio, para los habitantes de la comuna, no es el primer medio de comunicación, si es el tercero, por tanto es factible de considerar que existe una vinculación entre ambos aspectos.

Es así que en momentos de gestión de eventos extremos es factible apreciar la convergencia en la red de gobernanza en Frutillar. Sus funcionarios claramente expresaron que ante cualquier inconveniente su red inicia en lo local, para luego ir a la escala provincial, regional e incluso nacional. De tal red coordinada, bajan los recursos y acciones para mitigar la crisis. En tal modelo debilidades claramente existen, señalan que no es factible el acceso a recursos financieros autónomos para enfrentar una crisis. Éstos son entregados y previamente ejecutados en razón a políticas públicas que no están en sintonía con las particularidades del territorio y que solo ejecutan planes y/o acciones desarrolladas fuera del municipio.

En el caso de las organizaciones locales, estas se caracterizan por reunirse con periodicidad, cuentan con intereses en resolver problemas comunes, pero solo asisten pocos a las reuniones, la poca asistencia contribuye a la poca fluidez de la información para solución de problemas lo que lleva a que los integrantes de las organizaciones consideren que no están alcanzando metas colectivas. Cabe destacar que no fue posible de identificar, ni en los habitantes ni en los funcionarios municipales el rol de las organizaciones dentro del sistema de gobernanza ambiental de la comuna. Informados por el conocimiento en profundidad de la comuna y la municipalidad, a nuestro modo de ver las organizaciones tienen algunas desventajas de articulación principalmente producto por la falta de retornos, si no hay un resultados, difícilmente 
existirá la motivación para participar a nivel comunitario para la resolución de problemas.

Entonces, si la literatura afirma que el capital social y al gobernanza son factores decisivos para la adaptación al cambio climático ¿Por qué observamos debilidades en las estrategias de adaptación en la comuna de Frutillar? Creemos que aquello tiene que ver con falta de motivación y el escaso interés de desplegar estrategias de adaptación desde las autoridades locales. Tal como se mencionó anteriormente, en las actas de consejo municipal no fue posible advertir alguna mención sobre realizar estrategias de adaptación a eventos extremos, solo se mencionaba en agenda actividades tendientes a la mitigación coyuntural de un problema. Si bien, desde los funcionarios públicos existe una incomodidad de aplicar estrategias de mitigación que no están contextualizadas en la comuna, aquello no fue mencionado por las autoridades en tales actas y menos plasmado en las actividades desplegadas en el territorio.

\section{Conclusión}

Claramente las actividades locales de mitigación de un evento climático extremo no son suficientes, abordan una dimensión del problema, y solo sirven para un momento y temas puntuales, por ejemplo repartir agua durante la sequía. La adaptación es más integral y compleja. Si bien en ciertos contextos son necesarias y urgentes las medidas de mitigación, jamás deberían reemplazar a las estrategias de adaptación.

Es así que respuestas locales al cambio climático y/o eventos extremos son fundamentales para la adaptación, ésta no solo se consigue con la buena voluntad o con los instrumentos de planificación sensibles al tema o las políticas y programas nacionales para la mitigación, es fundamental el desarrollo de prácticas efectivas que se basen en información fidedigna y contextualizadas desde y para los territorios, que a su vez, sean desplegadas a través de la participación de distintos actores territoriales y que sean ejecutadas en coordinación con los distintos actores de la red local, regional, nacional e internacional de gobernanza. Sin esa perspectiva, la adaptación al cambio climático podría llegar a ser un fracaso.

Las evidencias que tenemos del caso de la comuna de Frutillar revelan la necesidad de fortalecer los procesos de gobernanza y el capital social. Asimismo, revelan la necesidad de incluir otros temas en relación a la adaptación. Pensando en nuestra propia estrategia futura de investigación, por ejemplo, importante sería 
incluir en la agenda de investigación análisis comparados y explicativos, no solo exploratorios, de las estrategias de la reducción de riesgos de desastres, las infraestructuras críticas, y la protección medioambiental a nivel local. Por el momento, con la presentación de un caso no es posible generar demasiados elementos para la comparación, y la discusión sobre otros contextos territoriales.

Karla V. Romero Baeza é Mestre em Ciências Sociais e Doutoranda de Ciências Sociais em Estudos Territoriais da Universidad de Los Lagos. E-mail: kromerobaeza.87@gmail.com.

\section{Referências}

ABDÓN, Luis; MEZA, Francisco. Cambio Climático: consecuencias y desafíos para Chile. Centro Interdisciplinario de Cambio Global. v. 3, n. 9. Universidad Católica de Chile. 2008. Disponível em: <http://politicaspublicas.uc.cl/wp-content/uploads/2015/02/cambio-climatico-consecuentas-ydesafios-paras-chile.pdf>. Acesso em: 06 abr. 2014.

AGUILAR, Luis. El aporte de la Política Pública y de la Nueva Gestión Pública a la gobernanza. Reforma y Democracia. n. 39. Caracas. 2007. Disponível em: <http://www.bibliotecajb.org/Portals/0/docs/Maestrias/Alta_Direccion_Publica/2.\%20La\%20Nuev a\%20Gestion\%20Publica\%20(Luis\%20Aguilar).pdf>. Acesso: 06 abr. 2014.

AHN, T.K.; OSTROM, Elionor. Social Capital and Collective Action. In: CATIGLIONE, Dario et al. (Orgs.). The Handbook of Social Capital. Oxford University Press, 2008.

ANDERSSON, Krister; VALDIVIESO, Patricio. Why local governments matter: Adapting to a changing climate in Chile. Harvard Rev. Lat. Am. v. 18, n. 3, p. 68-82, 2018.

BLANCO, Ismael; GOMÀ, Ricard. La Crisis del Modelo de Gobierno Tradicional. Reflexiones en torno a la governance participativa y de proximidad. Gestión y política pública. v. 12, n. 1, 2003. Disponível em: <http://www.plataformademocratica.org/Publicacoes/11505.pdf>. Acesso em: 15 abr. 2014.

CEPAL. La Economía del Cambio climático en América Latina y el Caribe. Santiago de Chile. 2008. Disponível em: <http://www.cepal.org/publicaciones/xml/8/38128/SintesisEconomia_cambio_climatico-WEB.pdf>. Acesso em: 15 abr. 2018.

COLEMAN, James. Social Capital in the Creation of Human Capital. The American Journal of Sociology. v. 94, 1998. Disponível em: <http://onemvweb.com/sources/sources/social_capital.pdf>. Acesso em: 06 maio 2014.

COMISIÓN DE LAS COMUNIDADES EUROPEAS. La gobernanza europea. Un libro Blanco. 2001. Disponível em: <http://www.uclg-decentralisation.org/sites/default/files/whitepapergover nanceeu_2001_es.pdf>. Acesso em: 06 maio 2014. 
CONAMA. Estudio de la variabilidad climática en Chile para el Siglo XXI. Universidad de Chile. 2006. Disponível em: <http://dgf.uchile.cl/PRECIS/articles-39442_pdf_Estudio_texto.pdf>. Acesso em: 01 abr. 2014.

ESSER, Hartmut. The two meanings of Social Capital. In: CATIGLIONE, Dario et al. (Orgs.). The Handbook of Social Capital. Oxford University Press, 2008, p. 22 - 49.

FAO. Planificación de la adaptación al CC en base comunitario. 2009. Disponível em: <http://www.webgeo.de/fao-webgeo-2-intro_spa/>. Acesso em: 10 jun. 2014.

GALINDO, Luis; SAMANIEGO, José Luis; ALATORRE, José Eduardo; FERRER, Jimy; REYES, Orlando. Paradojas y riesgos del crecimiento económico en América Latina y el Caribe. Una visión ambiental de largo plazo. Serie Medioambiente y Desarrollo. CEPAL. 2013. Disponível em: <https:/www.cepal.org/publicaciones/xml/4/53514/Paradojasyriesgosdelcrecimiento.pdf>. Acesso em: 22 jun. 2015.

GALINDO, Luis; SAMANIEGO, José Luis; ALATORRE, José Eduardo; FERRER, Jimy; REYES, Orlando. Procesos de adaptación al cambio climático. Análisis de América Latina. Estudios del Cambio Climático en América Latina. CEPAL. Santiago de Chile. 2014. Disponível em: < https://repositorio.cepal.org/bitstream/handle/11362/37613/S1500005_es.pdf?sequence=1>. Acesso em: 14.abr. 2015.

IPCC. First Assesment Report. Working Group I: Scientific Assessment of Climate Change. 1990. Disponível em: $<$ http://www.ipcc.ch/publications_and_data/publications_ipcc_first_assessment_1990_wg1.shtml>. Acesso em: 30 mar. 2014.

. Third Assessment Report: Climate Change 2001. Synthesis Report. 2001. Disponível em: <http://www.grida.no/publications/other/ipcc_tar/>. Acesso em: 30 mar. 2014.

. Fourth Assessment Report: Climate Change 2007. Contribution of Working Groups I, II and III to the Fourth Assessment Report of the Intergovernmental Panel on Climate Change. 2007. Disponível em: <http://www.ipcc.ch/publications_and_data/ar4/syr/en/contents.html>. Acesso em: 30 mar. 2014.

Climate Change: The Physical Science Basis. 2013. Disponível em: <http://www.ipcc.ch/report/ar5/wg1/>. Acesso em: 30 mar. 2014.

- Impactos, adaptación y vulnerabilidad. 2014. Disponível em: <https://www.ipcc.ch/ pdf/assessment-report/ar5/wg2/ar5_wgII_spm_es.pdf>. Acesso em: 30 mar. 2014.

LIN, Nan, A Network Theory of Social Capital. In: CATIGLIONE, Dario et al. (Orgs.). The Handbook of Social Capital. Oxford University Press, 2008, p. 22 - 49.

MINISTERIO DE MEDIO AMBIENTE. Plan Nacional de Medio Ambiente. Chile. 2014. Disponível em: <http://portal.mma.gob.cl/wp-content/uploads/2014/12/PAN-web2.pdf>. Acesso em: 10 mar. 2015.

MUNICIPALIDAD DE FRUTILLAR. Actas de Consejo Municipal Desde enero de 2011 a mayo de 2014. 2014.

MUNICIPALIDAD DE FRUTILLAR. Catálogo organizaciones comunitarias Frutillar. 2018.

MUNICIPALIDAD DE FRUTILLAR. Plan de Desarrollo Comunitario 2015 - 2018. 2015. Disponível em: < http://pladesfrutillar.cl/web/wp-content/uploads/2017/02/PLadeco-20152018.pdf/>. Acesso em: 30 jun. 2015. 
NATERA, Antonio. La noción de gobernanza como gestión pública participativa y reticular. Universidad Carlos III de Madrid. 2004. Disponível em: <http://earchivo.uc3m.es/handle/10016/590>. Acesso em: 10 jun. 2014.

OSTROM, Elionor. Institutions and the environment. Economic Affairs, v. 28, n. 3, p. 24-31, 2008. Disponível em: <https://onlinelibrary.wiley.com/doi/epdf/10.1111/j.1468-0270.2008.00840.x >. Acesso em: 25 abr. 2018.

PUTNAM, Robert. Making Democracy Work. Civic Traditions in Modern Italy. Princeton University Prest. 1993. Disponível em: <http://ebookbrowse.com/1255640943-making-democracywork-civic-traditions-in-modern-italy-1994-putnam-pdf-d106713078>. Acesso em: 10 mar. 2014.

PUTNAM, Robert. Bowling Alone: The Collapse and Revival of American Community. USA: Touchstone Books by Simon \& Schuster, 2000.

RHODES, Raw. The New Governance: Governing without Government. Political Studies. v. 44, 1996. Disponível em: <http://onlinelibrary.wiley.com/doi/10.1111/j.1467-9248.1996.tb01747.x/ abstract>. Acesso em: 10 mar. 2014.

ROTHSTEIN, Bo; STOLLE, Dietlind. Political Institutions and Generalized Trust. In: CATIGLIONE, Dario et al. (Orgs.). The Handbook of Social Capital, Oxford University Press, 2008, p.273-303.

USLANER, Eric Trust. Democracy and Governance: Can government policies influence generalized trust? In: HOOGHE, Marc; STOLLE, Dietlind (Orgs.). Generating Social Capital. Civil Society and institutions comparative perspective. USA: Palgrave Macmillan, 2003.

VALDIVIESO, Patricio. Políticas Públicas de participación ciudadana y CS en Chile. Revista Debates. v. 1, n. 6, 2012. Disponível em: <http://seer.ufrgs.br/debates/article/download/26117/16875>. Acesso em: 10 mar. 2014.

Municipal governance, environmental management and disaster risk reduction in Chile". Bulletin of Latin American Research. v. 36, p. 440-458, 2016. Disponível em: <https://www.researchgate.net/publication/320032729_Municipal_Governance_Environmental_M anagement_and_Disaster_Risk_Reduction_in_Chile>. Acesso em: 19 abr. 2018.

. Facilitadores institucionales y sociales para la gobernanza local de los riesgos ambientales. Análisis empírico con municipios chilenos. Opin. Publica. v. 23, n. 3, p. 538-579, 2017. Disponível em: <http://www.scielo.br/scielo.php?script=sci_arttext\&pid=S0104-62762017000300538>. Acesso em: 19 abr. 2018.

VALDIVIESO, Patricio; ANDERSSON, Krister. Local politics of environmental disaster risk management: institutional analysis and lessons from Chile. Journal of Environment \& Development. v. 26, n. 1, p. 51-81, 2017. Disponível em: <https://www.researchgate.net/ publication/312120988_Local_Politics_of_Environmental_Disaster_Risk_Management_Institution al_Analysis_and_Lessons_From_Chile>. Acesso em: 19 abr. 2018.

VALDIVIESO, Patricio; ANDERSSON, Krister; VILLENA-ROLDÁN, Benjamín. Institutional drivers of adaptation in local government decision-making: evidence from Chile. Climatic Change, v. 143, p. 157-171, 2017. Disponível em: <https://www.researchgate.net/publication/315815751_ Institutional_Drivers_of_Adaptation_in_Local_Government_Decision_Making_Evidence_from_C hile>. Acesso em: 19 abr. 2018.

VALDIVIESO, Patricio; DAVIDOVICS, Gabriel. Enfoques y metodologías para la investigación empírica del capital social. Santiago: Universidad Central. 2016. 
VALDIVIESO, Patricio; VILlENA-ROLDAN, Benjamín. Participation in Organizations, Trust, and Social Capital Formation: Evidence from Chile. Departamento de Ingeniería Industrial, Universidad de Chile. 2012. Disponível em: <http://www.dii.uchile.cl/wp-content/uploads/2012/11/ Participation-in-Organizations-Trust-and-Social-Capital-Formation-Evidence-from-Chile-PatricioValdivieso-y-Benjamin-Villena-Roldan.pdf>. Acesso em: 30 jun. 2014.

. Opening the Black Box of Social Capital Formation. American Political Science Research.v. 108, n. 1, p. 121-143, 2014. Disponível em: <https:/www.cambridge.org/core/journals/americanpolitical-science-review/article/opening-the-black-box-of-social-capital-

formation/BA61754708A28ABC4D81BA5C71572696>. Acesso em: 19 abr. 2018.

WHITTINGHAM, María Victoria. ¿Qué es la gobernanza y para qué sirve? Revista de análisis intencional. v. 2, n. 10, p. 219-235, 2010. Disponível em: <http://revistas.utadeo.edu.co/ index.php/RAI/article/view/24/26>. Acesso em: 19 abr. 2014.

Texto recebido em 03 de junho de 2018. Aprovado em 10 de agosto de 2018. 
